\title{
2000000 Steiner Triple Systems of Order 19
}

\author{
By D. R. Stinson and H. Ferch
}

\begin{abstract}
Using a hill-climbing algorithm, we construct 2117600 Steiner triple systems of order 19. These are tested for isomorphism by means of invariants, and 2111276 are shown to be nonisomorphic.
\end{abstract}

1. Introduction. Let $v$ be a positive integer. A Steiner triple system of order $v$ (denoted $\operatorname{STS}(v))$ is a pair $(X, \mathscr{B})$, where $X$ is a set of $v$ elements (called points), and $\mathscr{B}$ is a set of $v(v-1) / 63$-subsets of $X$ (called blocks), such that every pair of points occurs in a unique block. It is easily seen that $v \equiv 1$ or 3 modulo 6 is a necessary condition for the existence of an STS $(v)$; Kirkman [4] in 1847 demonstrated the sufficiency of this condition.

Two $\operatorname{STS}(v),\left(X_{1}, \mathscr{B}_{1}\right)$ and $\left(X_{2}, \mathscr{B}_{2}\right)$, are said to be isomorphic if there is a bijection $\phi: X_{1} \rightarrow X_{2}$ such that $\{x, y, z\} \in \mathscr{B}_{1}$ if and only if $\{\phi(x), \phi(y), \phi(z)\} \in$ $\mathscr{B}_{2}$. The number of nonisomorphic $\operatorname{STS}(v)$ is denoted by $N(v)$. It is known that $N(1)=N(3)=N(7)=N(9)=1, N(13)=2$, and $N(15)=80$. Results concerning $N(19)$ include enumerations of particular classes of STS(19). For example there are precisely 4 nonisomorphic cyclic STS(19) [1], 10 nonisomorphic 2-rotational STS(19) [5], and 184 nonisomorphic reverse STS(19) [3]. STS(19) containing subsystems have also been studied. Let $(X, \mathscr{B})$ be an $\operatorname{STS}(v)$. A subsystem of order $w$ (denoted sub-STS $(w))$ is a pair $(Y, \mathscr{C})$, where $Y \subseteq X$ and $\mathscr{C} \subseteq \mathscr{B}$, which is an $\operatorname{STS}(w)$ in its own right. An STS(19) can contain subsystems of orders 1 or 3 (trivially), 7, or 9. In [12], it was shown that there are at least 2450 nonisomorphic STS(19) which contain 3 sub-STS(7) intersecting in a point. Also, in [2] and [11], it was shown that there are exactly 284457 nonisomorphic STS(19) containing a sub-STS(9). Such STS(19) are also studied in [6].

2. An Improved Lower Bound for $N(19)$. From Section $1, N(19) \geqslant 284457$. It is the purpose of this note to improve this bound for $N(19)$. We accomplish this by constructing STS(19) using a hill-climbing algorithm described in [9]. The algorithm is nondeterministic, so we hope that STS constructed by it are random in some sense. Although there is no proof known that this algorithm will succeed, it appears in practice to require time $O\left(v^{2} \log v\right)$ to construct an $\operatorname{STS}(v)$. Programmed in PASCAL/VS and run on the University of Manitoba Amdahl 470/V8 computer, STS(19) are constructed, on average, in $.021 \mathrm{sec}$.

Thus, we have a practical method of generating many STS(19), most of which we hope will be nonisomorphic. We tested isomorphism by means of invariants. An

Received September 14, 1983; revised April 20, 1984 and August 15, 1984

1980 Mathematics Subject Classification. Primary 05B05. 
invariant is a function, defined on the set of all STS, such that isomorphic STS have the same invariants (the converse need not hold). We desire invariants to be fast, effective, and compact. We used two invariants, which are described in [10]: fragments and trains. For STS(19), the two invariants require 74 bytes of memory. They can be calculated in an average time of $.026 \mathrm{sec}$.

In our study of STS(19) we are also interested in subsystems, so we checked each STS(19) for the presence of sub-STS(7) and sub-STS(9). This can be done in about $.004 \mathrm{sec}$.

As each STS(19) was generated, we stored the relevant information on tape: the random seed used to generate the system, the two invariants, and two Boolean flags indicating the presence of subsystems. We thus have an 80 byte record for each STS(19), and the total time required is about $.05 \mathrm{sec}$. We filled one 2400 foot tape with the above information. 2117600 STS(19) were constructed, in about 30 hours of CPU time.

The second step was to sort the records, using the 74 bytes of the invariants as a key. This sort was accomplished in $2 \mathrm{~min}$. $45 \mathrm{sec}$. Finally, we made one pass through the sorted records ( $1 \mathrm{~min} .19 \mathrm{sec}$.), obtaining the results of Table 1.

TABLE 1

2117600 Steiner triple systems of order 19

\begin{tabular}{cr} 
property & number found \\
\cline { 2 - 2 } distinct invariants & 2111276 \\
distinct invariants & \\
and no sub-STS(9) & 2111230 \\
sub-STS(7) & 14196 \\
sub-STS(9) & 46 \\
sub-STS(7) and sub-STS(9) & 2
\end{tabular}

Thus we have the following lower bound.

ThEOREM. $N(19) \geqslant 2395687$.

Proof. There are exactly 284457 nonisomorphic STS(19) which contain a subSTS(9), and at least 2111230 which do not.

3. An Estimate for $N(19)$. What is the true value of $N(19)$ ? We can obtain one estimate, based on the fact $R=2111276$ distinct invariants were found in a sample of size $S=2117600$. The estimated population is the solution $N$ to the equation $(1-R / N)=(1-1 / N)^{S}$ (see, for example, [8]). This equation can easily be solved by Newton's method, giving $N=3.54 \times 10^{8}$.

Although $N$ is huge, it is probably too low, for two reasons. First, the population we are estimating is that of invariants, and not nonisomorphic STS(19). It can happen, and does, that nonisomorphic STS(19) have the same invariants. We know of no way to estimate the probability of this occurring. Second, we construct STS(19) by means of a pseudo-random number generator with a period of $2^{30}$. So our population is restricted to those $2^{30}\left(\approx 10^{9}\right) \mathrm{STS}(19)$ which can be constructed from the particular pseudo-random number generator we used. 
We can obtain an estimate in another fashion, by considering the STS(19) with a sub-STS(9). We have noted that there are exactly 284457 such STS. Of the 2117600 STS we constructed, 46 contained a sub-STS(9). Taking ratios, we get an estimate of $N=284457 / 46 \times 2117600=1.309 \times 10^{9}$. It may be that our hill-climbing algorithm is less likely to produce STS(19) containing sub-STS(9) than a truly random algorithm; so this estimate may be too high. Nevertheless, we suspect that the true value of $N(19)$ is approximately $10^{9}$.

We have an observation to make concerning subsystems. An $\operatorname{STS}(v)$ is said to be planar if it contains no sub-STS( $w)$ with $w>3$. In [7], Quackenbush conjectures that almost all STS are planar. Our results provide some empirical evidence in favor of this conjecture. Of the 80 STS(15), 23 are nonplanar, which is $29 \%$. Of the STS(19) we constructed, only 14240 were nonplanar, which is $.67 \%$.

4. Summary. In summary, we would like to emphasize the applicability of hill-climbing techniques to the study of Steiner triple systems. We believe hill-climbing is particularly useful for obtaining information concerning random designs; indeed, it is the only practical method known at present.

Department of Computer Science

University of Manitoba

Winnipeg, Manitoba, Canada R3T 2N2

1. S. BAYS, "Recherche des systèmes cycliques de triples de Steiner différents pour $N$ premier (ou puissance de nombre premier) de la forme $6 n+1$," J. Math. Pures Appl. (9), v. 2, 1923, pp. 73-98.

2. R. Deherder, Recouvrements, Thesis, Université Libre de Bruxelles, 1976, 212 pp.

3. R. H. F. Denniston, “Non-isomorphic reverse Steiner triple systems of order 19," Ann. Discrete Math., v. 7, 1980, pp. 255-264.

4. T. P. Kirkman, “On a problem in combinations," Cambridge and Dublin Math. J., v. 2, 1847. $191-204$.

5. K. T. Phelps \& A. Rosa, “Rotational Steiner triple systems," Discrete Math., v. 33, 1981, pp. $57-66$.

6. A. R. Prince, "Steiner triple systems of order 19 constructed from the Steiner triple system of order 9." Proc. Roy. Soc. Edinburgh Sect. A., v. 94, 1983, pp. 89-92.

7. R. W. QUackenbuSh, “Algebraic speculations about Steiner systems," Ann. Discrete Math., v. 7 , 1980, pp. 25-35.

8. G. A. F. SEBER, "The Estimation of Animal Abundance and Related Parameters, Griffin, London and High Wycombe, 1982.

9. D. R. Srinson, "Hill-climbing algorithms for the construction of combinatorial designs," Ann. Discrete Math. (To appear.)

10. D. R. Stinson "A comparison of two invariants for Steiner triple systems: fragments and trains," Ars Combin., v. 16, 1983, pp. 69-76.

11. D. R. Stinson \& E. SEAH, “284457 Steiner triple systems of order 19 contain a subsystem of order 9." (Preprint)

12. R. M. Wilson, “Non-isomorphic Steiner triple systems," Math. Z., v. 135, 1974, pp. 303-313. 